\title{
Thermo-Kinetic Model of Burning for Pyrolyzing Materials
}

\author{
STANISLAV I. STOLIAROV ${ }^{1}$ and RICHARD E. LYON ${ }^{2}$ \\ ${ }^{1}$ SRA International, Inc., 3120 Fire Road, Egg Harbor Township, NJ 08234, USA \\ ${ }^{2}$ FAA W. J. Hughes Technical Center, Atlantic City International Airport, NJ 08405, USA
}

\begin{abstract}
One of the main obstacles to development of more effective passive fire protection for transportation is the lack of a quantitative understanding of the relations between the results of various materials fire tests used in this field. The need for multiple testing techniques arises from the complexity of fire phenomena and their sensitivity to environmental conditions. This work addresses this problem by developing a computational tool that predicts the behavior of materials exposed to fire. While it is not expected that this tool will eliminate the need for fire testing, the goal is to considerably reduce the number and complexity of the tests necessary for a comprehensive characterization of the materials of interest. The foundation of this tool is a mathematical model that describes transient thermal energy transport, chemical reactions, and transport of gases through the condensed phase. The model also captures such important aspects of material's behavior as charring and intumescence. This paper provides a detailed description of the onedimensional version of this model and summarizes results of its verification.
\end{abstract}

KEYWORDS: pyrolysis, combustion, polymer, modeling

\section{NOMENCLATURE LISTING}

\begin{tabular}{llll}
$T$ & temperature & $v$ & \multicolumn{2}{l}{ convection coefficient } \\
$C$ & heat capacity & $f$ & radiative heat flux \\
$m$ & mass & $a$ & absorbance \\
$V$ & volume & Greek & \\
$h$ & heat of reaction & $\rho$ & density \\
$r$ & rate of reaction & $\gamma$ & swelling factor \\
$q$ & rate of heat transfer & $\theta$ & stoichiometric coefficient \\
$k$ & thermal conductivity & $\lambda$ & gas transfer coefficient \\
$S$ & surface area & $\varepsilon$ & emissivity \\
$j$ & rate of mass transfer & $\alpha$ & absorption coefficient \\
$P$ & pressure & $\Phi$ & energy density \\
$t$ & time & &
\end{tabular}

\section{INTRODUCTION}

A substantial number of studies have been dedicated to development of mathematical models that describe degradation of solid materials exposed to external heat flux. These models range in their complexity from analytical formulations based on assumption of a steady-state [1,2] to complete, numerical solutions of the transient heat and mass transport coupled with chemical reactions [3,4]. Most of these models are onedimensional. They predict the rate of mass loss from a unit area of the exposed surface. Some models incorporate a description of the gas-phase combustion. Such models are frequently used to simulate the spread of flame [5,6] (flame spread models are usually two-dimensional). Potentially, these models can be employed to understand and predict materials flammability. However, their utilization has been hampered by reliance on assumptions that make each of them applicable to a relatively narrow range of conditions and limited number of materials.

The purpose of this work is to develop a versatile computational tool that is capable of modeling pyrolysis and combustion of a wide range of polymeric materials including composites. Our approach is called thermo-kinetic model or ThermaKin because it combines transient thermal energy transport and material transformations described by means of chemical kinetics. The model also includes a description of transport of gases through the condensed phase and tracks changes in the volume of material. The complexity of the model can be manipulated by introducing or removing material components, which are characterized by temperature-dependent physical and chemical properties. At this stage, the one- 
dimensional version of this model has been completed. This version simulates conditions encountered in bench-scale fire calorimeters $[7,8]$. It is equipped with flexible boundary conditions that include time dependent radiative and convective heat fluxes and possible in-depth absorption of the radiation. Surface ignition is simulated by altering the heat fluxes when a specified mass flux of decomposition products is reached.

\section{MODEL DESCRIPTION}

\section{Components and Reactions}

In ThermaKin, material is represented by a mixture of components. Every component is characterized by density, heat capacity, thermal conductivity, gas transfer coefficient, emissivity, and absorption coefficient. The first 4 properties in this list are defined by a flexible function of temperature ( $T)$,

property $=p_{0}+p_{1} T+p_{n} T^{n}$

where $p_{0}, p_{1}, p_{n}$, and $n$ are user-specified parameters. Emissivity and absorption coefficient are defined by constants. All components are divided into 3 categories: solids, liquids, and gases. This categorization is used in the calculation of the volume of material as explained below.

The heat capacity $(c)$ of a given amount of material is calculated as

$c=\sum_{i=1}^{N_{c}} m_{i} c_{i}$

where $m_{i}$ and $c_{i}$ are mass and heat capacity of the $i$-th component; and $N_{c}$ is the number of components. The volume of material $(V)$ is defined by

$V=\sum_{s=1}^{N_{s}} \frac{m_{s}}{\rho_{s}}+\sum_{l=1}^{N_{l}} \frac{m_{l}}{\rho_{l}}+\gamma \sum_{g=1}^{N_{g}} \frac{m_{g}}{\rho_{g}}$

where $\rho$ is component density. Subscripts $s, l$, and $g$ are used to refer to solid, liquid, and gaseous components, respectively. Swelling factor $\gamma$, which may assume a value between 0 and 1 , describes reaction of the volume of material to the presence of gases. When $\gamma=0$, the presence of gases has no effect on the volume. When $\gamma=1$, gases contribute to the volume of material in accordance with their densities. $\gamma$ is calculated by volume-weighted averaging of the swelling factor specified for solids $\left(\gamma_{s}\right)$ and liquids $\left(\gamma_{1}\right)$ :

$\gamma=\frac{\gamma_{s} \sum_{s=1}^{N_{s}} \frac{m_{s}}{\rho_{s}}+\gamma_{l} \sum_{l=1}^{N_{l}} \frac{m_{l}}{\rho_{l}}+\tau \sum_{g=1}^{N_{g}} \frac{m_{g}}{\rho_{g}}}{\sum_{s=1}^{N_{s}} \frac{m_{s}}{\rho_{s}}+\sum_{l=1}^{N_{l}} \frac{m_{l}}{\rho_{l}}+\tau \sum_{g=1}^{N_{g}} \frac{m_{g}}{\rho_{g}}}$

$\tau$ is a parameter that is used to insure that, at the limit of very high gas content, the volume of material is defined by the densities of the gases.

Components may undergo reactions. Each reaction may have 1-2 reactants and 0-2 products:

$\theta_{1}$ COMP1 $+\theta_{2}$ COMP2 $\rightarrow \theta_{3}$ COMP3 $+\theta_{4}$ COMP $4+h$

The rate of reaction $(r)$ taking place in a unit volume of material is defined by

$r=A \exp \left(-\frac{E}{R T}\right)\left[\frac{m_{\text {СOMP } 1}}{V}\right]\left[\frac{m_{\text {СOMP } 2}}{V}\right]$

where $A$ and $E$ are the Arrhenius pre-exponential factor and activation energy. $R$ is the gas constant. In the absence of the second reactant, $m_{\text {COMP2 }}=V$. The rate of consumption/formation of a reactant/product is calculated by multiplying $r$ by the corresponding stoichiometric coefficient $(\theta)$. The rate of production of 
heat is calculated by multiplying $r$ by the heat of reaction $(h) . h$ is defined by the same type of temperature dependence as that used for component properties (see equation 1). The reaction description also includes specification of a lower or upper temperature limit. If temperature decreases below the lower limit or increases above the upper limit, the rate of reaction is set to 0 . Application of this limit increases computational efficiency (reaction rates are evaluated only at the temperatures where they are important) and facilitates usage of reactions for the description of phase transitions.

\section{Heat and Mass Transfer}

The conduction of heat is described by Fourier's law:

$q=-k S \frac{\partial T}{\partial x}$

$q$ is the rate of heat transfer; $S$ is the surface area across which the heat is transferred; and $x$ is the coordinate normal to this surface. $k$ is the thermal conductivity of material. It depends on relative amounts and spatial distribution of components. If components are stacked in uniform layers that are normal to the direction of the heat flow, the thermal conductivity is

$$
k_{n}=\frac{V}{\sum_{i=1}^{N_{c}} \frac{V_{i}}{k_{i}}}
$$

where $k_{i}$ and $V_{i}$ are thermal conductivity and volume contribution of the $i$-th component. If the layers are parallel to the direction of the heat flow, the thermal conductivity is

$$
k_{p}=\frac{\sum_{i=1}^{N_{c}} k_{i} V_{i}}{V}
$$

The $k_{n}$ and $k_{p}$ expressions can be derived using an analogy with electric circuits consisting of resistors connected in series and parallel [9].

For an arbitrary spatial distribution of components, the exact analytical expression of the thermal conductivity is not available. However, under the assumption that components do not affect thermal conductivities of each other, equations 8 and 9 provide lower and upper limits for the value of $k$. This means that the thermal conductivity of material can be represented as

$$
k=\beta k_{p}+(1-\beta) k_{n}
$$

where $\beta$ is a parameter that may assume a value between 0 and 1 . In ThermaKin, this representation is used in conjunction with the assumption that a pyrolyzing material can be characterized by a single value of $\beta$.

The transfer of mass is assumed to be driven by a concentration gradient:

$$
j_{g}=-\rho_{g} \lambda S \frac{\partial\left(\frac{\left(m_{g} / \rho_{g}\right)}{V}\right)}{\partial x}
$$

where $j_{g}$ is the rate of transfer of gas $g$ (only gaseous components are considered to be mobile). $\lambda$ is the gas transfer coefficient of material. It is calculated from the corresponding component coefficients using the same approach as that utilized for thermal conductivity (see equations 8-10). Note that $\lambda$ does not depend on the nature of the gas that is being transferred (i.e., on volumetric basis, all gases subjected to the same concentration gradient are transferred with the same rate).

Application of Boyle's law, which states that the product of the pressure and volume of a fixed amount of gas is constant, transforms equation 11 into 
$j_{g}=-\frac{\rho_{g} \lambda S}{P^{d e f}} \frac{\partial\left(\phi P_{g}\right)}{\partial x}$

$\phi$ is the volume fraction of material occupied by gases; $P_{g}$ is the partial pressure of gas $g$; and $P^{\text {def }}$ is the pressure at which the gas density $\left(\rho_{g}\right)$ is defined. If material is rigid and does not expand with addition of gases (i.e., $\gamma=0$ and $\phi$ is constant), equation 12 assumes the form of Darcy's law, which is used to describe the flow of fluids through porous media [10]. On the other hand, if material expands proportionally to the volume of added gases (i.e., $\gamma>0$ and pressure inside the material is constant), equation 12 states that the flow of gas is driven by the gradient of its volumetric fraction.

\section{Conservation Equations}

The overall behavior of a pyrolyzing object is described by mass and energy conservation equations. In ThermaKin, these equations are formulated in terms of rectangular finite elements. Each element is characterized by component masses and temperature. The formulation (described below) contains the following assumptions. The heat exchange between transferred gases and the rest of material is instantaneous. The energy associated with the bulk velocity of gases and the work of expansion/contraction of material are negligible.

Let us consider an object consisting of 2 elements, L and R, shown in Fig. 1. An application of the law of conservation of mass to the $g$-th component in element $\mathrm{R}$ yields

$\frac{\Delta m_{g}^{\mathrm{R}}}{\Delta t}=V^{\mathrm{R}} \sum_{j=1}^{N_{r}} \theta_{j}^{g} r_{j}^{\mathrm{R}}+\lambda^{\mathrm{LR}} \rho_{\mathrm{g}}^{\mathrm{LR}} S \frac{\left(\frac{\left(m_{g}^{\mathrm{L}} / \rho_{\mathrm{g}}^{\mathrm{L}}\right)}{V^{\mathrm{L}}}-\frac{\left(m_{g}^{\mathrm{R}} / \rho_{\mathrm{g}}^{\mathrm{R}}\right)}{V^{\mathrm{R}}}\right)}{\Delta x}$

where $\Delta m_{g}{ }^{\mathrm{R}}$ is the change in the component mass during the time $\Delta t$. The terms on the right hand side of the equation are contributions from reactions and mass transfer from element $L$ (the mass transfer term is present only if component $g$ is a gas). $\theta_{j}^{g}$ is the stoichiometric coefficient in front of component $g$ in the $j$-th reaction. This coefficient is set to be negative when the component is a reactant and positive when it is a product. LR superscript is used to refer to averages of the parameters obtained for each of the two elements. $\Delta x$ is the distance between the centers of the elements (see Fig. 1).

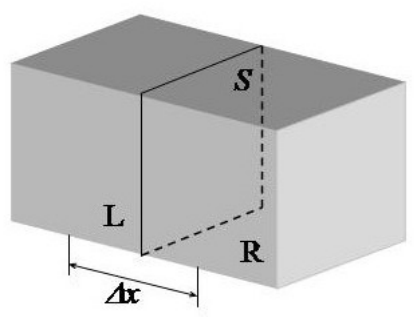

Fig. 1. Two-Element Object

An application of the law of conservation of energy to element $R$ yields

$c^{\mathrm{R}} \frac{\Delta T^{\mathrm{R}}}{\Delta t}=V^{\mathrm{R}} \sum_{j=1}^{N_{r}} h_{j}^{\mathrm{R}} r_{j}^{\mathrm{R}}+k^{\mathrm{LR}} S \frac{\left(T^{\mathrm{L}}-T^{\mathrm{R}}\right)}{\Delta x}+\frac{1}{2} \sum_{g=1}^{N_{g}} c_{g}^{\mathrm{LR}}\left(T^{\mathrm{L}}-T^{\mathrm{R}}\right) j_{g}^{\mathrm{LR}}$

where $\Delta T^{\mathrm{R}}$ is the change in the element temperature during the time $\Delta t$. The terms on the right hand side of the equation account for heat generation by reactions, and conduction and convection of heat from element $\mathrm{L} . \mathrm{j}_{g}^{\mathrm{LR}}$ is the rate of flow of gas $g$ from L to R (a detailed expression of this rate is given by the last term in equation 13).

The description provided by equations 13 and 14 is expanded to more complex systems in a straightforward manner. Addition of an element adjacent to element $\mathrm{R}$ results in addition of the terms that correspond to the 
flows of matter and energy from this element to the equations. To make this description complete, the conservation equations should be written for every component and every element of a pyrolyzing object and complemented by the conditions at the boundaries. The accuracy of the description strongly depends on the choice of element size and time step $(\Delta t)$. This dependence is analyzed below.

\section{Boundary Conditions}

The one-dimensional version of ThermaKin has 2 boundaries, which can be viewed as top and bottom surfaces of a flat material object. Conditions at the boundaries are comprised of a description of mass and heat transfer through the boundary surfaces. Each of the boundaries is described separately using the following mathematical framework. The mass flow $\left(j_{i}^{\mathrm{B}}\right)$ of component $i$ from the boundary element $\mathrm{B}$ is expressed as

$$
j_{i}^{\mathrm{B}}=\left\{\begin{array}{l}
a_{i} \rho_{i}^{\mathrm{B}} S^{\mathrm{B}}\left(\frac{\left(m_{i}^{\mathrm{B}} / \rho_{i}^{\mathrm{B}}\right)}{V^{\mathrm{B}}}-b_{i}\right) \\
\text { or } \\
a_{i} S^{\mathrm{B}} \exp \left(-\frac{b_{i}}{R T^{\mathrm{B}}}\right)
\end{array}\right.
$$

$S^{\mathrm{B}}$ is the surface area of the boundary; $a_{i}$ and $b_{i}$ are user-specified parameters; and $R$ is the gas constant. The primary function of the linear expression is to remove/introduce gases from/to a pyrolyzing material. The exponential expression can be employed to simulate a surface reaction (such as oxidation) or dripping.

Convective heat flow $\left(q_{c}^{\mathrm{B}}\right)$ into the boundary element is defined by

$$
\begin{aligned}
& q_{c}^{\mathrm{B}}=v S^{\mathrm{B}}\left(T^{\mathrm{e}}-T^{\mathrm{B}}\right) \\
& T^{\mathrm{e}}=T_{0}^{\mathrm{e}}+T_{t}^{\mathrm{e}} t
\end{aligned}
$$

where $v$ is the convection coefficient and $T^{\mathrm{e}}$ is the temperature outside of material. This temperature can be a constant or a linear function of time $(t)$. Radiative heat flow $\left(q_{r}^{\mathrm{A}}\right)$ into material is expressed as

$$
\begin{aligned}
& q_{r}^{\mathrm{A}}=\varepsilon^{\mathrm{A}} S^{\mathrm{B}}\left(f-\sigma\left(T^{\mathrm{A}}\right)^{4}\right) \\
& f= \begin{cases}f_{10}+f_{1 t} t & t \leq t_{1} \\
f_{20}+f_{2 t} t & t_{1}<t \leq t_{2}\end{cases}
\end{aligned}
$$

$\varepsilon^{A}$ is emissivity of the element that absorbs radiation (element A). $\sigma$ is the Stefan-Boltzmann constant. $f$ designates the flux of external radiation. It is defined by a sequence of 2 linear time dependencies, which can be specified to be periodic (i.e., the flux history can be repeated with the period of $t_{2}$ ).

The element that absorbs radiation is determined at every time step using either maximum absorption or random absorption algorithm. In both cases, the external radiation is assumed to penetrate material in the direction normal to the boundary surface and behave in accordance with Beer-Lambert law [11]:

$$
f^{\mathrm{A}}=f^{\mathrm{I}} a^{\mathrm{A}} l^{\mathrm{A}}
$$

where $f^{\mathrm{A}}$ is the flux entering element $\mathrm{A}$ and $f^{\mathrm{A}}$ is the flux absorbed by the element. $a^{\mathrm{A}}$ is absorbance and $l^{\mathrm{A}}$ is thickness of the element. When the maximum absorption algorithm is employed, the element that, according to equation 18, absorbs most of the radiation is assumed to absorb all of it. In the case of the random absorption algorithm, the absorbing element is selected at random using the Beer-Lambert distribution of absorbed energy as a probability density guiding this selection. In both approaches, the absorbing element also acts as a gray body reflector and emitter (see equation 17). The emissivity and absorbance used in equations 17 and 18 are calculated from component emissivities $\left(\varepsilon_{i}\right)$ and absorption coefficients $\left(\alpha_{i}\right)$ : 


$$
\varepsilon=\frac{\sum_{i=1}^{N_{c}} \varepsilon_{i} V_{i}}{V}
$$

$$
a=\frac{\sum_{i=1}^{N_{c}} \alpha_{i} m_{i}}{V}
$$

The ThermaKin boundary conditions are also equipped with a sub-model simulating surface ignition. The criterion for the ignition (CI) is based on component mass fluxes:

$$
\mathrm{CI}=\sum_{i=1}^{N_{c}} \frac{\left(j_{i}^{\mathrm{B}} / S^{\mathrm{B}}\right)}{\xi_{i}}
$$

where $\xi_{i}$ is a user-specified critical mass flux of component $i$. When CI is above 1 , the flame is turned on. The radiative heat flux from the flame, defined by a constant, is added to the external heat flux $f$. At the same time, convective heat transfer parameters $v$ and $T^{e}$ are replaced by constant parameters describing the flaming conditions.

\section{Solution Methodology}

The conservation equations formulated above can be cast into the following general form:

$y_{i}^{t+\Delta t}=y_{i}^{t}+F_{i}\left(y_{i}, y_{j}, y_{k}, \ldots\right) \Delta t$

where $y_{i}^{t+\Delta t}$ is component mass or temperature in some element $\mathrm{E}$ at the time $t+\Delta t . y_{i}^{t+\Delta t}$ is unknown and needs to be determined from the value of this parameter $\left(y_{i}^{t}\right)$ at the current time $(t)$ and the rate, $F_{i}$, with which this parameter changes. $F_{i}$ is a function of component masses and temperatures $\left(y_{i}, y_{j}, y_{k}, \ldots\right)$ in element $\mathrm{E}$ and adjacent elements. $F_{i}$ also implicitly depends on time and can be approximated by the average of its values at $t$ and $t+\Delta t$ :

$y_{i}^{t+\Delta t}=y_{i}^{t}+\frac{\left[F_{i}\left(y_{i}^{t}, y_{j}^{t}, y_{k}^{t}, \ldots\right)+F_{i}\left(y_{i}^{t+\Delta t}, y_{j}^{t+\Delta t}, y_{k}^{t+\Delta t}, \ldots\right)\right]}{2} \Delta t$

This approach is called the Crank-Nicolson scheme [12].

Equation 23 written for every component mass and temperature of every element forms a set of coupled algebraic equations. These equations are nonlinear in nature and, therefore, are difficult to solve. To rectify this problem, function $F_{i}$ at $t+\Delta t$ is linearized by using the first 2 terms of the Taylor series:

$F_{i}^{t+\Delta t}=F_{i}^{t}+\frac{\partial F_{i}^{t}}{\partial y_{i}}\left(y_{i}^{t+\Delta t}-y_{i}^{t}\right)+\frac{\partial F_{i}^{t}}{\partial y_{j}}\left(y_{j}^{t+\Delta t}-y_{j}^{t}\right)+\frac{\partial F_{i}^{t}}{\partial y_{k}}\left(y_{k}^{t+\Delta t}-y_{k}^{t}\right)+\ldots$

Substitution of the linearized function into equation 23 produces a system of linear equations that has block tridiagonal character. These equations are solved (to obtain $y_{i}^{t+\Delta t}, y_{j}^{t+\Delta t}, y_{k}^{t+\Delta t}, \ldots$ ) using an approach similar to LU decomposition with iterative improvement of the solution [12]. Solving these equations constitutes performance of an integration time step. This solution procedure has been implemented using the ANSI/ISO C++ and its standard library.

\section{MODEL VERIFICATION}

The ability of ThermaKin to predict experimental observations will be tested in future studies. In this work, the key submodels of ThermaKin have been verified by comparing the results of numerical calculations with analytical solutions. The heat transfer submodel has been tested against analytical solutions for conduction in a semi-infinite-solid and in-depth absorption of radiation. The reactions submodel has been 
checked against an analytical solution for 4 coupled first and second order reactions. The mass transfer submodel has been verified against an analytical solution for diffusion from a thin layer.

\section{Conduction in Semi-Infinite Solid}

The temperature $(T)$ of a semi-infinite-solid that is heated at the boundary surface by convection can be expressed [9] as

$$
\begin{aligned}
& \frac{T-T^{\mathrm{i}}}{T^{\mathrm{e}}-T^{\mathrm{i}}}=1-\operatorname{erf}\left(\frac{x}{2 \sqrt{X}}\right)-\exp \left(\frac{v x}{k}+\frac{v^{2} X}{k^{2}}\right)\left(1-\operatorname{erf}\left(\frac{x}{2 \sqrt{X}}+\frac{v \sqrt{X}}{k}\right)\right) \\
& X=\frac{k}{\rho c} t
\end{aligned}
$$

where $T^{\mathrm{i}}$ is the initial temperature of the solid; and $T^{\mathrm{e}}$ is the temperature outside of the solid. For $\rho=1000$ $\mathrm{kg} \mathrm{m}^{-3}, c=2000 \mathrm{~J} \mathrm{~kg}^{-1} \mathrm{~K}^{-1}, k=0.2 \mathrm{~W} \mathrm{~m}^{-1} \mathrm{~K}^{-1}, v=90 \mathrm{~W} \mathrm{~m}^{-2} \mathrm{~K}^{-1}, T^{\mathrm{i}}=300 \mathrm{~K}$, and $T^{\mathrm{e}}=900 \mathrm{~K}$, this expression is plotted in Fig. 2 together with the results of ThermaKin calculations (performed using identical set of properties and conditions). A four centimeter $(0.04 \mathrm{~m})$ thick slab of material with no heat exchange at one of the boundaries was used in ThermaKin to simulate the infinite solid. The element size and time step were set at $2 \times 10^{-5} \mathrm{~m}$ and $0.05 \mathrm{~s}$, respectively. A factor of 5 increase in either of these integration parameters produced no noticeable changes in the results. With the exception of a small discrepancy between $t=450 \mathrm{~s}$ curves, the analytical and ThermaKin temperatures are indistinguishable. The discrepancy is a consequence of a limited accuracy of the spreadsheet program that was used to calculate the analytical temperatures.

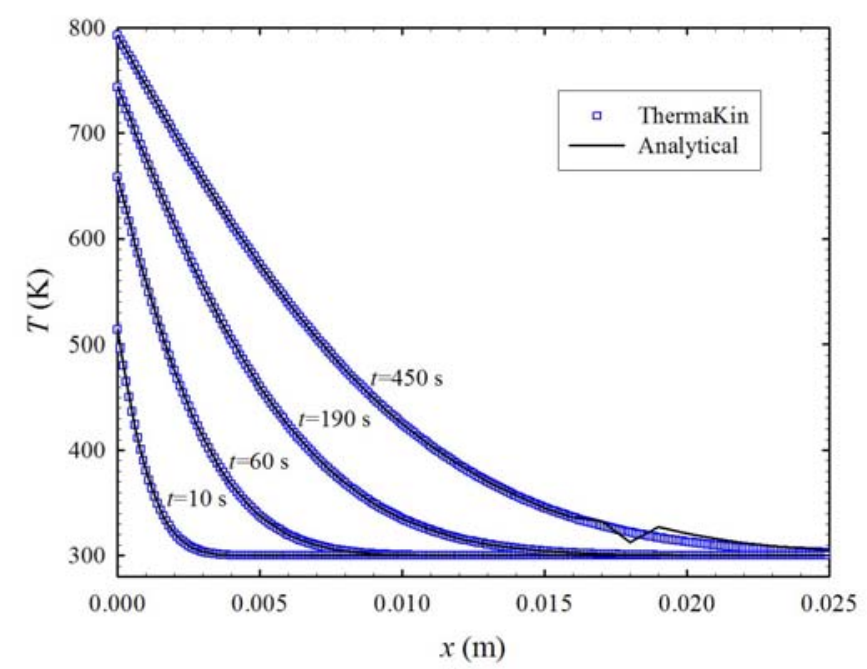

Fig. 2. Comparison of Analytical and ThermaKin Solutions for Conduction in Semi-Infinite Solid

\section{In-Depth Absorption of Radiation}

According to Beer-Lambert law [11], the density of energy $(\Phi)$ delivered into a material by radiation during a period of time $t$ can be calculated as

$\Phi=f^{\mathrm{i}} \alpha \rho \exp (-\alpha \rho x) t$

where $f$ is the radiative heat flux that penetrates the surface of the material. To determine whether the random absorption algorithm (described above) provides an adequate description of the radiative energy transfer, ThermaKin calculations were performed on a $0.003 \mathrm{~m}$ thick layer of material exposed to $5 \times 10^{4} \mathrm{~W}$ $\mathrm{m}^{-2}$ radiative heat flux. The boundary conditions were formulated in such a way that all radiation was absorbed and none of it was reemitted. The material was characterized by $\rho=1000 \mathrm{~kg} \mathrm{~m}^{-3}, c=2000 \mathrm{~J} \mathrm{~kg}^{-1}$ $\mathrm{K}^{-1}$, and $\alpha=3 \mathrm{~m}^{2} \mathrm{~kg}^{-1}$. The thermal conductivity was set to a negligibly small value $\left(1 \times 10^{-10} \mathrm{~W} \mathrm{~m}^{-1} \mathrm{~K}^{-1}\right)$ to 
prevent non-radiative energy transfer. The simulations were run for $10 \mathrm{~s}$ using 0.05 and $0.001 \mathrm{~s}$ time steps. The element size was set at $2 \times 10^{-5} \mathrm{~m}$.

The energy densities obtained from the ThermaKin simulations are compared with those calculated using equation 26 in Fig. 3. The ThermaKin energy densities were obtained by multiplying increases in local temperatures (achieved as a result of $10 \mathrm{~s}$ exposure) by the product of $\rho$ and $c$. The large time step simulation produces a noisy energy pattern because of a small number of times (200) the Beer-Lambert distribution is sampled. When the number is increased to 10000 (the small time step simulation), ThermaKin energy distribution becomes closely aligned with the analytical result.

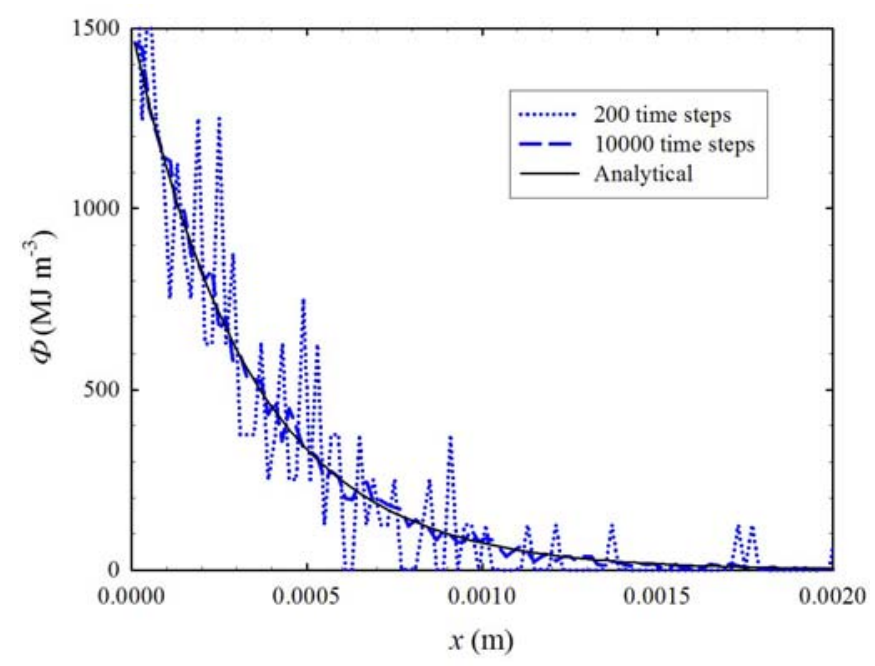

Fig. 3. Comparison of Analytical and ThermaKin Distributions of Absorbed Radiative Energy

\section{Chemical Reactions}

Let us consider the following set of chemical reactions:

$1 \mathrm{C} \rightarrow 1 \mathrm{PC}$

$0.5 \mathrm{C}+0.5 \mathrm{C} \rightarrow 1 \mathrm{PCC}$

$1 \mathrm{D} \rightarrow 1 \mathrm{PD}$

$1 \mathrm{D}+0 \mathrm{C} \rightarrow 1 \mathrm{PD}$

$\mathrm{C}$ and $\mathrm{D}$ are reactants. PC, PCC, and PD are products. The reactions are assumed to obey the rate law formulated above (equations 5 and 6). The temperature of the system is set to be constant. Note that, while $\mathrm{C}$ is not consumed in reaction 30, the rate of this reaction depends on the amount of $\mathrm{C}$ (in accordance with equation 6). Under these conditions, a straightforward integration of the rate equations becomes possible:

$$
\begin{aligned}
& {[\mathrm{C}]_{t}=\frac{[\mathrm{C}]_{0} r k_{27} \exp \left(-r k_{27} t\right)}{[\mathrm{C}]_{0} r k_{28}\left(1-\exp \left(-r k_{27} t\right)\right)+r k_{27}}} \\
& {[\mathrm{PC}]_{t}=[\mathrm{PC}]_{0}+\frac{r k_{27}}{r k_{28}} \ln \left([\mathrm{C}]_{0} \frac{r k_{28}}{r k_{27}}\left(1-\exp \left(-r k_{27} t\right)\right)+1\right)} \\
& {[\mathrm{PCC}]_{t}=[\mathrm{PCC}]_{0}+[\mathrm{C}]_{0}-[\mathrm{C}]_{t}-[\mathrm{PC}]_{t}+[\mathrm{PC}]_{0}}
\end{aligned}
$$

$[\mathrm{D}]_{t}=[\mathrm{D}]_{0} \exp \left(-r k_{29} t\right)\left(\frac{r k_{27}}{[\mathrm{C}]_{0} r k_{28}\left(1-\exp \left(-r k_{27} t\right)\right)+r k_{27}}\right)^{\frac{r k_{30}}{r k_{28}}}$ 
$[\mathrm{PD}]_{t}=[\mathrm{PD}]_{0}+[\mathrm{D}]_{0}-[\mathrm{D}]_{t}$

Square brackets are used to indicate initial (subscript 0) or current (subscript $t$ ) concentrations of components. $r k_{27}, r k_{28}, r k_{29}$, and $r k_{30}$ are rate constants of the corresponding reactions. Each rate constant represents the product of the pre-exponential factor and exponent in equation 6.

For $[\mathrm{C}]_{0}=990 \mathrm{~kg} \mathrm{~m}^{-3},[\mathrm{D}]_{0}=10 \mathrm{~kg} \mathrm{~m}^{-3},[\mathrm{PC}]_{0}=[\mathrm{PCC}]_{0}=[\mathrm{PD}]_{0}=0, r k_{27}=0.02 \mathrm{~s}^{-1}, r k_{28}=1 \times 10^{-4} \mathrm{~m}^{3} \mathrm{~kg}^{-1} \mathrm{~s}^{-}$ ${ }^{1}, r k_{29}=0.002 \mathrm{~s}^{-1}$, and $r k_{30}=5 \times 10^{-5} \mathrm{~m}^{3} \mathrm{~kg}^{-1} \mathrm{~s}^{-1}$, analytically calculated concentration histories are plotted in Fig. 4. ThermaKin solution obtained for identical conditions using a single isolated element to represent the chemical system is also shown in the figure. The results of the ThermaKin calculations (performed using $0.05 \mathrm{~s}$ time step) are in excellent agreement with the analytical solution.
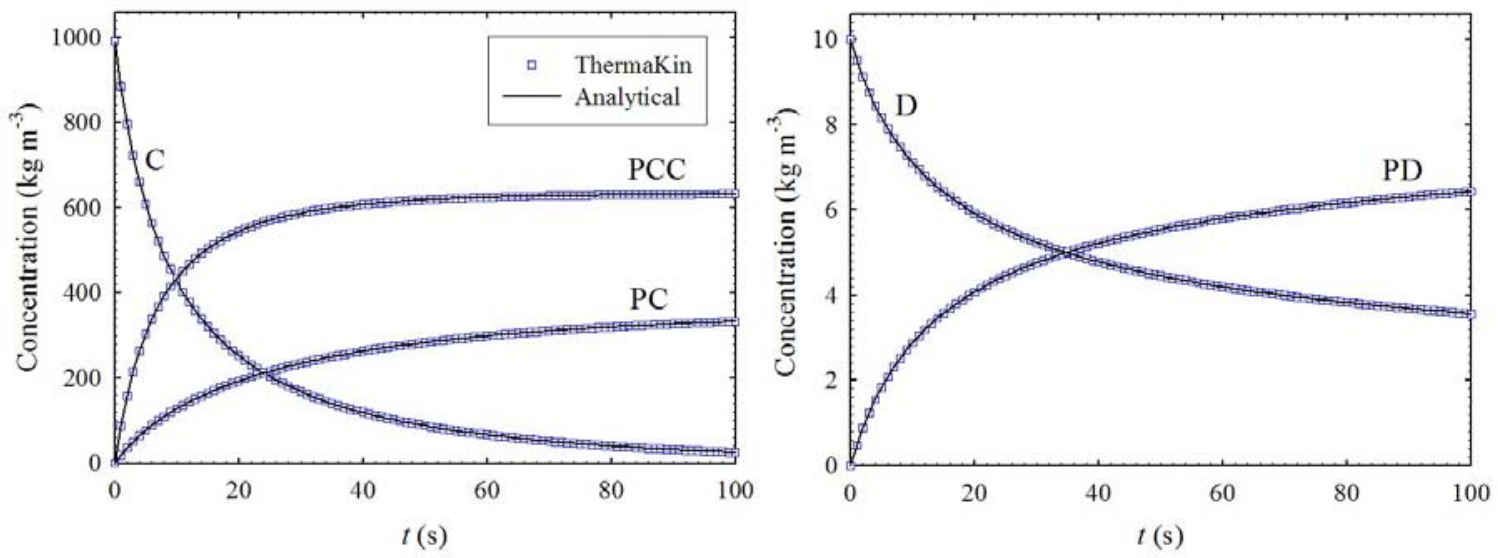

Fig. 4. Comparison of Analytical and ThermaKin Solutions for a System of Chemical Reactions

\section{Diffusion from a Thin Layer}

When component $\mathrm{C}$ is introduced into a very thin layer of material that consists of component $\mathrm{D}$, the subsequent one-dimensional diffusion of $\mathrm{C}$ can be described [13] by

$$
[\mathrm{C}]=\frac{M_{\mathrm{C}}}{2 \sqrt{\pi d_{\mathrm{CD}} t}} \exp \left(-\frac{\left(x-x_{\mathrm{C}}^{\mathrm{i}}\right)^{2}}{4 d_{\mathrm{CD}} t}\right)
$$

where [C] is the concentration of $\mathrm{C}$; and $M_{\mathrm{C}}$ is the total amount of $\mathrm{C}$ per unit area. $d_{\mathrm{CD}}$ is the diffusion coefficient of $\mathrm{C}$ in $\mathrm{D} . x_{\mathrm{C}}^{\mathrm{i}}$ is the position of the thin layer. A ThermaKin model of this process was comprised of $0.03 \mathrm{~m}$ thick slab of solid component $\mathrm{D}$ held at a constant temperature with no mass transfer at the boundaries. Two hundred and twenty grams per square meter $\left(0.22 \mathrm{~kg} \mathrm{~m}^{-2}\right)$ of gaseous component $\mathrm{C}$ was placed into a single element located in the center of the slab. The swelling factor for solid components $\left(\gamma_{s}\right)$ was set to 0 . This means that only component D (the density of which was set at $1000 \mathrm{~kg} \mathrm{~m}^{-3}$ ) contributed to the material's volume. The gas transfer coefficient for $\mathrm{D}\left(\lambda_{\mathrm{D}}\right)$, which is, under these conditions, equivalent to the diffusion coefficient $d_{\mathrm{CD}}$, was set at $8 \times 10^{-8} \mathrm{~m}^{2} \mathrm{~s}^{-1}$.

A comparison of the results of the ThermaKin calculations with those obtained using equation 36 is shown in Fig. 5. The ThermaKin results, which were computed using $2 \times 10^{-5} \mathrm{~m}$ element size and $0.001 \mathrm{~s}$ time step, are in excellent agreement with the analytical solution. A factor of 5 increase in either of the integration parameters produced no noticeable changes in the results. 


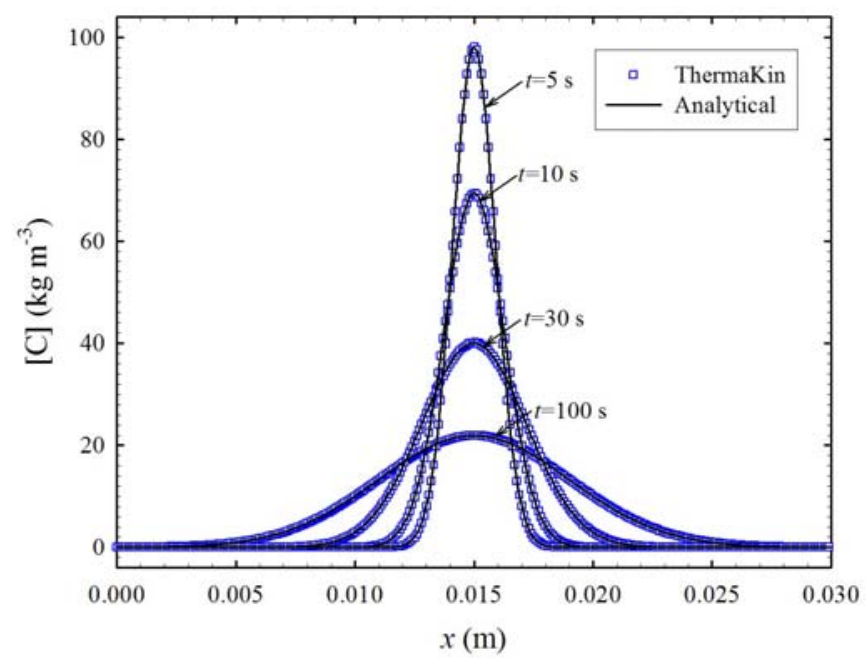

Fig. 5. Comparison of Analytical and ThermaKin Solutions for Diffusion from a Thin Layer

\section{COMPUTATIONAL COSTS}

The computational cost of a ThermaKin calculation depends on the size of a material object, length of simulation, and choice of integration parameters. The parameter values are dictated by the scales of the processes that need to be resolved. The goal of the analysis described below was to determine element size, time step, and the amount of computer resources that provide the converged solution for what could be considered as a typical one-dimensional pyrolysis problem. All calculations (including those described above) were performed on a PC equipped with a $3.4 \mathrm{GHz}$ Intel Xeon (single core) processor, $2 \mathrm{~GB}$ of RAM, and Windows XP operating system. The ThermaKin program was compiled using Microsoft Visual $\mathrm{C}++$.NET non-optimizing compiler.

The material used in these calculations was defined by the following properties: $\rho=1000 \mathrm{~kg} \mathrm{~m}^{-3}, c=$ $500+3 T \mathrm{~J} \mathrm{~kg}^{-1} \mathrm{~K}^{-1}, k=0.2 \mathrm{~W} \mathrm{~m}^{-1} \mathrm{~K}^{-1}$, and $\varepsilon=0.95$. Decomposition of this material was assumed to occur in a single step first order endothermic reaction. The Arrhenius pre-exponential factor, activation energy, and heat of reaction were set at $5 \times 10^{15} \mathrm{~s}^{-1}, 2.5 \times 10^{5} \mathrm{~J} \mathrm{~mol}^{-1}$, and $-1 \times 10^{6} \mathrm{~J} \mathrm{~kg}^{-1}$, respectively. The stoichiometric coefficient of the reactant (the material) was equal to 1 . No product was specified. The products of the decomposition were assumed to leave the material instantaneously.

One side of $0.005 \mathrm{~m}$ thick layer of this material, which was initially at $300 \mathrm{~K}$, was irradiated by $5 \times 10^{4} \mathrm{~W} \mathrm{~m}$ ${ }^{2}$ heat flux. Application of the maximum absorption algorithm and a high absorption coefficient $\left(1000 \mathrm{~m}^{2}\right.$ $\mathrm{kg}^{-1}$ ) insured that the radiation was absorbed at the surface. The convective heat transfer at the surface was defined by $T^{\mathrm{e}}=300 \mathrm{~K}$ and $v=10 \mathrm{~W} \mathrm{~m}^{-2} \mathrm{~K}^{-1}$ (see equation 16). The other side of the material layer was thermally insulated. This was achieved by turning off external heat fluxes and adding a boundary element consisting of a non-degradable material with $k=1 \times 10^{-10} \mathrm{~W} \mathrm{~m}^{-1} \mathrm{~K}^{-1}$ and $\varepsilon=0$. All mass transfer was turned off.

For $1 \times 10^{-5} \mathrm{~m}$ element size and $0.01 \mathrm{~s}$ time step, the rate of mass loss, which is probably the most important descriptor of a pyrolysis process, is shown in Fig. 6. An order of magnitude increase in element size produces no significant changes in the rate history, which means that for this range of element sizes the solution is converged. Further increase in element size to $5 \times 10^{-4} \mathrm{~m}$ yields a diverged solution (see Fig. 6). The results of a similar analysis applied to time step are depicted in Fig. 7. With element size fixed at $1 \times 10^{-}$ ${ }^{5} \mathrm{~m}$, the integration diverges when time step is increased to $2 \mathrm{~s}$. When element size is set at a higher value of $1 \times 10^{-4} \mathrm{~m}$, the divergence does not occur until time step reaches $20 \mathrm{~s}$. On the basis of these analyses, it can be concluded that a combination of $3 \times 10^{-5} \mathrm{~m}$ element size and $0.1 \mathrm{~s}$ time step should be more than sufficient to produce a converged solution for this type of pyrolysis problem. A four hundred second simulation performed using these integration parameters required about $30 \mathrm{~s}$ of the computer time. 


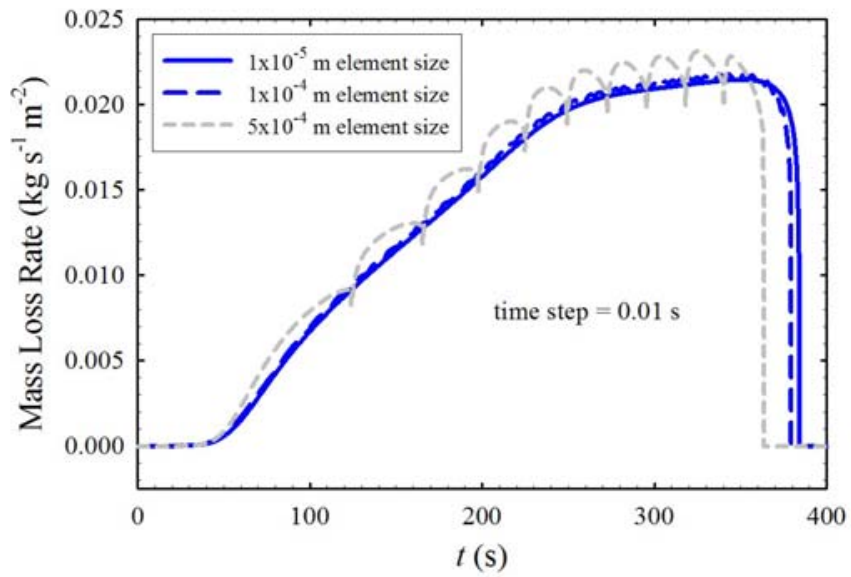

Fig. 6. Dependence of Mass Loss Rate History on Element Size

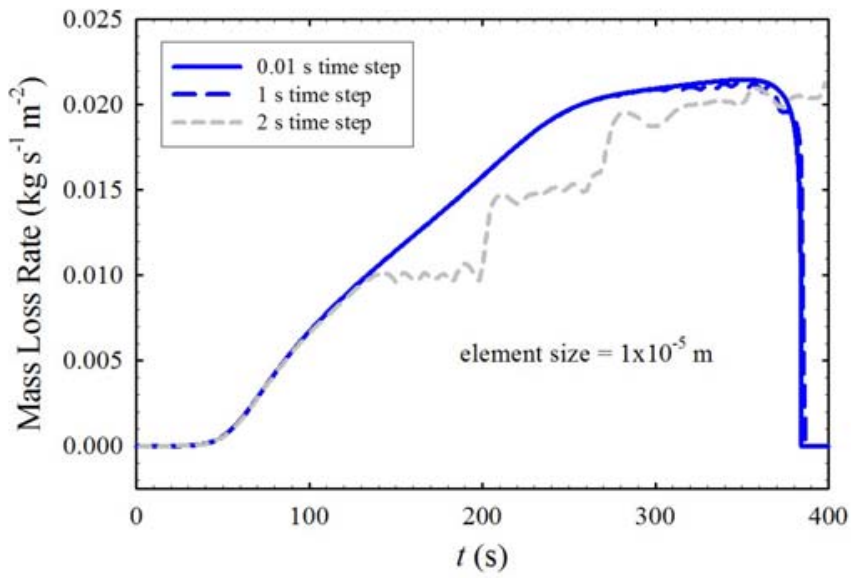

Fig. 7. Dependence of Mass Loss Rate History on Time Step

\section{CONCLUSIONS}

A versatile numerical model (called ThermaKin) of pyrolysis and combustion of polymeric materials has been formulated. The one-dimensional version of this model has been implemented as a $\mathrm{C}++$ program and verified by comparing its results with analytical solutions. The computational costs of pyrolysis simulations performed using this program were found to be low. The future work will be focused on verification of the ability of ThermaKin to reproduce experimental observations (in particular, cone calorimetry experiments [7] performed on non-charring and charring plastics) and on identifying the key properties that define the materials fire behavior.

\section{REFERENCES}

[1] Staggs J. E. J., “A Theory for Quasi-Steady Single-Step Thermal Degradation of Polymers,” Fire and Materials, vol. 22, pp. 109-118, $1998 . \quad$ doi:10.1002/(SICI)10991018(1998050)22:3<109::AID-FAM643>3.0.CO;2-C

[2] Lyon R. E., “Heat Release Kinetics,” Fire and Materials, vol. 24, pp. 179-186, 2000. doi:10.1002/1099-1018(200007/08)24:4<179::AID-FAM736>3.0.CO;2-V

[3] Di Blasi C., "Analysis of Convection and Secondary Reaction Effects within Porous Solid Fuels Undergoing Pyrolysis," Combustion Science and Technology, vol. 90, pp. 315-340, 1993. doi:10.1080/00102209308907620 
[4] Staggs J. E. J., “A Simple Model of Polymer Pyrolysis Including Transport of Volatiles,” Fire Safety Journal, vol. 34, pp. 69-80, 2000. doi:10.1016/S0379-7112(99)00043-0

[5] Agrawal S., Atreya A., "Wind-Aided Flame Spread over an Unsteadily Vaporizing Solid," Twenty-Fourth Symposium (International) on Combustion, pp. 1685-1693, 1992.

[6] Di Blasi C., "Processes of Flames Spreading over the Surface of Charring Fuels: Effects of the Solid Thickness," Combustion and Flame, vol. 97, pp. 225-239, 1994. doi:10.1016/00102180(94)90006-X

[7] ASTM Standard E 1354-04, "Standard Test Method for Heat and Visible Smoke Release Rates for Materials and Products Using an Oxygen Consumption Calorimeter," American Society for Testing and Materials, West Conshohocken, PA, 2004.

[8] ASTM Standard E 906-06, "Standard Test Method for Heat and Visible Smoke Release Rates for Materials and Products using a Thermopile Method," American Society for Testing and Materials, West Conshohocken, PA, 2006.

[9] Holman J. P., “Heat Transfer,” McGraw-Hill, Boston, MA, 2002.

[10] Scheidegger A. E., "The Physics of Flow through Porous Media," University of Toronto Press, Toronto, Canada, 1974.

[11] Atkins P. W., "Physical Chemistry,” W. H. Freeman and Company, San Francisco, CA, 1978.

[12] Press W. H., Teukolsky S. A., Vetterling W. T., Flannery B. P., "Numerical Recipes in C++: The Art of Scientific Computing,” Cambridge University Press, Cambridge, UK, 2002.

[13] Middleman S., "An Introduction to Mass and Heat Transfer: Principles of Analysis and Design,” John Wiley \& Sons, Inc., New York, NY, 1998. 